\title{
Profilaxis de enfermedad por virus de Epstein Barr en niños y adultos receptores de trasplante de órganos sólidos y de precursores hematopoyéticos
}

\author{
Paula Catalán y Andrea Alba
}

\section{Prophylaxis against Epstein Barr disease in pediatric and adult patients undergoing solid organ and hematopoietic stem cells transplantation}

Post transplant lymphoproliferative disease (PTLD) associated with EBV infection is one of the most lifethreatening complications in SOT and HSCT. Risk factors for infection or reactivation of EBV in SOT are the use of greater immunosuppression, seronegative receptor and CMV infection. In HSCT, the risk factors are related to type of transplant, HLA disparity, the greater immunosuppression, T-cell depletion and severe GVHD. There is no scientific evidence to support the use of specific therapy for prophylaxis of EBV infection. Prophylaxis recommendations focus on avoid exposure of transplant recipients to sources of virus, through hygiene practices such as hand washing (A3), avoid sharing utensils (B3) and avoid contact with potentially infected secretions (respiratory or saliva) (A2). For PTLD prevention, the recommendation is regular EBV viral load monitoring by rtPCR. In SOT with logarithmic rising of EBV loads, it is recommended to reduce immunosuppression and periodically perform exams to diagnose PTLD. In HSCT, it is recommended to reduce immunosuppression whenever possible, and use rituximab according to specific protocol. Acyclovir or gancyclovir have not proven to be of any efficacy in PTLD prophylaxis in SOT (C3) or HSCT (D2), so their administration as preemptive therapy is no recommended.

Key words: Epstein Barr virus, post transplant lymphoproliferative disease, prophylaxis, transplant, solid organ prophylaxis, hematopoietic stem cells transplantation.

Palabras clave: Virus de Epstein Barr, síndrome linfoproliferativo, post trasplante, profilaxis, trasplante, trasplante de órganos sólidos, trasplante de precursores hematopoyéticos.

\section{Epidemiología}

$\mathrm{E}$ 1 síndrome linfoproliferativo (SLP) asociado a virus de Epstein Barr (VEB) constituye una entidad de riesgo tanto en trasplante de órganos sólidos (TOS) como en trasplante de precursores hematopoyéticos (TPH). Históricamente, en la era pre-rituximab (anticuerpo monoclonal anti-CD20), el pronóstico era muy reservado con alta tasa de mortalidad, la que actualmente es de alrededor de 50\%. La incidencia global es de 3 a $15 \%$. La incidencia en TOS depende del órgano trasplantado: intestino y multivisceral (15-20\%), pulmón y corazón (10-15\%), hígado $(5-10 \%)$, riñón (1-5\%) mientras que en TPH la incidencia reportada es de 0,6 a $10 \%$, siendo mayor en la población pediátrica y en pacientes con factores de riesgo asociados ${ }^{1-6}$.

\section{Factores de riesgo para desarrollar un síndrome linfoproliferativo}

Trasplante de órganos sólidos. En TOS los factores de riesgo demostrados son: disparidad IgG VEB pretrasplante $\mathrm{D}(+) \mathrm{R}(-)$, TOS con primoinfección por VEB e infección por CMV.
Trasplante de precursores hematopoyéticos. Los factores de riesgo demostrados en TPH son el tipo de trasplante (haploidéntico y cordón mayor que trasplante familiar idéntico y éste, a su vez, mayor que autólogo), el uso de anticuerpos anti-linfocitos T (timoglobulina, OKT3), disparidad HLA, depleción de linfocitos $\mathrm{T}$ y

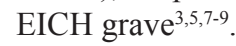

La literatura médica no registra evidencias para implementar medidas específicas de profilaxis en prevención de infección por VEB. Se recomienda evitar la exposición a fuentes del virus en los candidatos a trasplantes a través de prácticas de higiene tales como lavado de manos (A3), evitar el compartir utensilios (B3) y evitar el contacto con potenciales secreciones infectadas (respiratorias o saliva) (A2).

\section{Terapia anticipada}

En la última década se ha demostrado una clara relación entre carga viral de VEB (con técnica de RPC en tiempo real, RPC-TR-VEB) en ascenso y aparición de SLP, con un valor predictor negativo sobre $90 \%$, pero valor predictor positivo aún variable, debido a la disparidad
Unidad de Trasplante de Precursores Hematopoyéticos, Hospital Dr. Luis Calvo Mackenna, Santiago (PC). Unidad de Segunda Infancia, Hospital Dr. Luis Calvo Mackenna, Santiago (AA).

Los autores declaran no tener conflictos de interés.

Correspondencia a: paulacatalanm@gmail.com andreaalba@gmail.com 
de los estudios clínicos y la población estudiada ${ }^{1,4,10}$. En este escenario, se ha recomendado el monitoreo de carga viral de VEB por grupos de riesgo definido y la conducta anticipatoria. El monitoreo seriado y periódico se ha establecido como una conducta anticipatoria efectiva $a^{1,4,11,12}$. Los valores de corte para carga viral de VEB dependen de la técnica usada y el compartimento analizado (sangre total, plasma o leucocitos), siendo su determinación en sangre total la más estandarizada y usada en nuestro país.

Trasplante de órganos sólidos. En TOS se recomienda el seguimiento cada 2 a 4 semanas los primeros 6 meses, cada 2-3 meses hasta el año y cada 4 a 6 meses hasta los dos años post-trasplante, siempre ajustando la periodicidad a los factores de riesgo individuales. En caso de RPC-TR para VEB positiva sobre el valor de corte (receptor IgG (-): 1.000 copias/ml sangre total y receptor $\operatorname{IgG}(+): 10.000$ copias $/ \mathrm{ml}$ ) se debe repetir la determinación a los 3 a 7 días y, en caso de carga de VEB en ascenso logarítmico, se recomienda disminuir en lo posible la inmunosupresión (suspender anti-proliferativos y bajar niveles de anti-calcineurínicos a la mitad) y hacer evaluación clínica e imagenológica en busca del SLP (A2). En caso de no encontrarse evidencias de SLP, se recomienda mantener el monitoreo seriado de carga viral de VEB, pues se estima que 20 a $30 \%$ de estos pacientes desarrollarán un SLP en algún momento de su evolución. No se dispone de suficiente evidencia para recomendar rituximab como terapia anticipatoria o profilaxis en TOS con carga alta de VEB (C3) $)^{1,6}$.

Trasplante de precursores hematopoyéticos. En TPH se recomienda el seguimiento semanal con RPC-TRVEB hasta el día +100 , luego quincenal hasta los 6 meses post-TPH, manteniendo control mensual hasta el año y luego según su expresión clínica. En caso de RPC (+) (valor de corte: 1.000 copias $/ \mathrm{ml}$, criterio establecido en el Laboratorio de Biología Molecular del Hospital Luis Calvo Mackenna-U. de Chile), se repite la determinación. Al resultar negativa, el paciente debe continuar el seguimiento. En caso de positividad, debe reducirse la inmunosupresión en la medida que la condición clínica del paciente y la intensidad de EICH lo permita. Se recomienda indicar rituximab según protocolo ${ }^{13-17}(\mathrm{C} 3)$.

El uso de aciclovir o ganciclovir no han demostrado constituir medidas profilácticas efectivas en TOS (C3) ni en TPH (D2) ${ }^{18}$, no siendo recomendada su administración en esquemas de terapia anticipada (pre-emptive). Lo mismo ocurre con el uso de inmunoglobulina (C3) y LT citotóxicos específicos (TCD, UD, MMD) $)^{6,11,18,19}$.
Recomendación № 4: Profilaxis de enfermedad por virus de Epstein-Barr en trasplante de órganos sólidos y precursores hematopoyéticos

- Evitar la exposición a VEB de los candidatos a trasplante: lavado de manos (A3), evitar el compartir utensilios (B3), evitar contacto con potenciales secreciones infectadas (respiratorias o saliva) (A2)

- TOS: se recomienda seguimiento cada 2 a 4 semanas los primeros 6 meses, cada 2-3 meses hasta el año y cada 4 a 6 meses hasta los 2 años post-trasplante, siempre ajustando la periodicidad a los factores de riesgo individuales

- TPH: se recomienda el seguimiento semanal con RPC-TR-VEB hasta el día +100 , luego quincenal hasta los 6 meses post-TPH, manteniendo control mensual hasta el año y luego según manifestaciones clínicas

- En caso de RPC-TR-VEB (+), reducir la inmunosupresión en la medida que la condición clínica del paciente e intensidad de la EICH lo permita. Se recomienda indicar rituximab según protocolo (C3). El uso de aciclovir o ganciclovir no han demostrado constituir medidas profilácticas efectivas en TOS (C3) ni en TPH (D2)

\section{Resumen}

El síndrome linfoproliferativo (SLP) asociado a VEB constituye una grave complicación en TOS y en TPH. Los factores de riesgo de infección o reactivación de VEB en TOS son el uso de mayor inmunosupresión, la seronegatividad del receptor previa al trasplante y la infección por CMV. En TPH se consideran factores de riesgo el tipo de trasplante, disparidad HLA, mayor inmunosupresión, depleción linfocitaria y enfermedad injerto contra hospedero $(\mathrm{EICH})$ grave. No hay evidencia científica que apoye el uso de medidas específicas de profilaxis en prevención de infección por VEB. Se recomienda evitar la exposición a fuentes del virus de los candidatos a trasplantes a través de prácticas de higiene tales como lavado de manos (A3), evitar el compartir utensilios (B3) y evitar el contacto con potenciales secreciones infectadas (respiratorias $\mathrm{O}$ saliva) (A2). Para la prevención de SLP, se recomienda un esquema de monitoreo periódico de carga viral de VEB por RPC-TR. En el caso de TOS con cargas de VEB en ascenso logarítmico, se recomienda disminuir inmunosupresión y buscar activa y periódicamente la aparición de SLP. En TPH, se recomienda, en lo posible, disminuir la inmunosupresión y se reserva el uso de rituximab para casos específicos según protocolo. El uso de aciclovir o ganciclovir no han demostrado constituir medidas profilácticas efectivas en TOS (C3) ni en TPH (D2), no siendo recomendada su administración en esquemas de terapia anticipada. 


\section{Referencias bibliográficas}

1.- Allen U, Preiksaitis J. Epstein-Barr virus and posttransplant lymphoproliferative disorder in solid organ transplant recipients. Am J Transplant 2009; 9 Suppl 4: S87-96.

2.- Bhatia S, Ramsay N K, Steinbuch M, Dusenbery K E, Shapiro R S, Weisdorf D J, et al. Malignant neoplasms following bone marrow transplantation. Blood 1996; 87: 3633-9.

3.- Curtis R E, Travis L B, Rowlings P A, Socie G, Kingma D W, Banks P M, et al. Risk of lymphoproliferative disorders after bone marrow transplantation: a multiinstitutional study. Blood 1999; 94: 2208-16.

4.- Hoshino Y, Kimura H, Tanaka N, Tsuge I, Kudo K, Horibe K, et al. Prospective monitoring of the Epstein-Barr virus DNA by a real-time quantitative polymerase chain reaction after allogenic stem cell transplantation. Br J Haematol 2001; 115: 105-11.

5.- Cohen J, Gandhi M, Naik P, Cubitt D, Rao K, Thaker U, et al. Increased incidence of EBV-related disease following paediatric stem cell transplantation with reduced-intensity conditioning. Br J Haematol 2005; 129: 229-39.

6.- Nowalk A J, Green M. Epstein-Barr virusassociated posttransplant lymphoproliferative disorder: Strategies for prevention and cure. Liver Transpl 2010; 16: S54-S9.

7.- Gross T G, Steinbuch M, DeFor T, Shapiro R S, McGlave P, Ramsay N K, et al. B cell lymphoproliferative disorders following hematopoietic stem cell transplantation: Risk factors, treatment and outcome. Bone Marrow Transplant 1999; 23: 251-8.

8.- van Esser J W, van der Holt B, Meijer E, Niesters H G, Trenschel R, Thijsen S F, et al. Epstein-Barr virus (EBV) reactivation is a frequent event after allogeneic stem cell transplantation (SCT) and quantitatively predicts EBV-lymphoproliferative disease following T-cell-depleted SCT. Blood 2001; 98: 972-8.

9.- Landgren O, Gilbert E S, Rizzo J D, Socie G, Banks P M, Sobocinski K A, et al Risk factors for lymphoproliferative disorders after allogeneic hematopoietic cell transplantation. Blood 2009; 113: 4992-5001.

10.- Cesaro S, Murrone A, Mengoli C, Pillon M, Biasolo M A, Calore E, et al. The real-time polymerase chain reaction-guided modulation of immunosuppression enables the pre-emptive management of Epstein-Barr virus reactivation after allogeneic haematopoietic stem cell transplantation. Br J Haematol 2005; 128 : 224-33.

11.- DiNardo C D, Tsai D E. Treatment advances in posttransplant lymphoproliferative disease. Curr Opin Hematol 2010; 17: 368-74.

12.- Parker A, Bowles K, Bradley J A, Emery V, Featherstone C, Gupte G, et al. Diagnosis of post-transplant lymphoproliferative disorder in solid organ transplant recipients - BCSH and BTS Guidelines. Br J Haematol 2010; 149: 675-92.

13.- Gruhn B, Meerbach A, Hafer R, Zell R, Wutzler P, Zintl F. Pre-emptive therapy with rituximab for prevention of Epstein-Barr virus- associated lymphoproliferative disease after hematopoietic stem cell transplantation. Bone Marrow Transplant 2003; 31: 1023-5.

14.- Dominietto A, Tedone E, Soracco M, Bruno B, Raiola A M, Van Lint M T, et al. In vivo B-cell depletion with rituximab for alternative donor hemopoietic SCT. Bone Marrow Transplant 2011.

15.- Meijer E, Cornelissen J J. Epstein-Barr virus-associated lymphoproliferative disease after allogeneic haematopoietic stem cell transplantation: molecular monitoring and early treatment of high-risk patients. Curr Opin Hematol 2008; 15: 576-85.

16.- Styczynski J, Einsele H, Gil L, Ljungman P. Outcome of treatment of Epstein-Barr virusrelated post-transplant lymphoproliferative disorder in hematopoietic stem cell recipients: a comprehensive review of reported cases. Transpl Infect Dis 2009; 11: 383-92.

17.- Blaes A H, Cao Q, Wagner J E, Young J A, Weisdorf D J, Brunstein C G. Monitoring and preemptive rituximab therapy for Epstein-Barr virus reactivation after antithymocyte globulin containing nonmyeloablative conditioning for umbilical cord blood transplantation. Biol Blood Marrow Transplant 2010; 16: 287-91.

18.- Allen U, Green M. Prevention and treatment of infectious complications after solid organ transplantation in children. Pediatr Clinf North Am 2010; 57: 459-79

19.- Venturi C, Bueno J, Gavalda J, Tortola T, Pou L, Medina A, et al. Impact of valganciclovir on Epstein-Barr Virus polymerase chain reaction in pediatric liver transplantation: preliminary report. Transplant Proc 2009; 41: 1038-40. 